Kardiologe $2021 \cdot 15: 341-353$

https://doi.org/10.1007/s12181-021-00488-3

Online publiziert: 7. Juli 2021

(c) Deutsche Gesellschaft für Kardiologie -

Herz- und Kreislaufforschung e.V. Published

by Springer Medizin Verlag GmbH, ein Teil

von Springer Nature - all rights reserved 2021,

korrigierte Publikation 2021

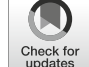

Angenommen: 26. Mai 2021

Christian Veltmann ${ }^{1}$ Joachim R. Ehrlich ${ }^{2}$. Ulrich M. Gassner ${ }^{3}$ Benjamin Meder $^{4}$. Martin Möckel ${ }^{5}$ Peter Radke ${ }^{6}$ Eberhard Scholz ${ }^{7}$ Hendrik Schneider ${ }^{8}$. Christoph Stellbrink ${ }^{9,10}$. David Duncker ${ }^{1}$

'Hannover Herzrhythmus Centrum, Klinik für Kardiologie und Angiologie, Medizinische Hochschule Hannover, Hannover, Deutschland

${ }^{2}$ Medizinische Klinik 1, St. Josefs Hospital, Wiesbaden, Deutschland

${ }^{3}$ Forschungsstelle für E-Health-Recht (FEHR), Universität Augsburg, Augsburg, Deutschland

${ }^{4}$ Klinik für Innere Med. III, Kardiologie, Angiologie und Pneumologie, Universitätsklinikum Heidelberg, Heidelberg, Deutschland

${ }^{5}$ Notfall- und Akutmedizin, Zentrale Notaufnahmen mit Chest Pain Units, Campus Mitte und Virchow, Charité - Universitätsmedizin Berlin, Berlin, Deutschland

${ }^{6}$ Klinik für Innere Medizin und Kardiologie, Schön Klinik Neustadt, Neustadt, Deutschland

${ }^{7}$ Innere Medizin I, GRN-Klinik Schwetzingen, Kardiologie, Schwetzingen, Deutschland

${ }^{8}$ Kanzlei für Wirtschafts- und Medizinstrafrecht, Wiesbaden, Deutschland

${ }^{9}$ Klinik für Kardiologie und Internistische Intensivmedizin, Klinikum Bielefeld gGmbH, Bielefeld, Deutschland

${ }^{10}$ Kommission für Klinische Kardiovaskuläre Medizin, Deutsche Gesellschaft für Kardiologie, Düsseldorf, Deutschland

\section{Wearable-basierte Detektion von Arrhythmien}

\section{Positionspapier der Deutschen Gesellschaft für Kardiologie}

\section{Präambel}

Die Digitalisierung in der Medizin hat dazu geführt, dass Körperfunktionen und primär medizinische Messwerte nicht nur mittels professioneller Geräte erhoben werden können, sondern dies inzwischen auch vom Laien durch von jedermann erwerbbare sog. Wearables möglich ist. Unter Wearables versteht man direkt am Körper oder der Kleidung getragene elektronische Geräte, die in der Lage sind, biophysikalische Daten zu erheben. Die bekanntesten Wearables sind Smartwatches, Armbänder und Brustgurte. Es existieren inzwischen Wearables in Form von Ringen, Brillen, Gürteln, Patches und T-Shirts (• Abb. 1). Wearables sind in der Lage, Parameter wie Schrittzahl, Körperposition, Kalori-

Aus Gründen der besseren Lesbarkeit und Verständlichkeit der Texte wird in SpringerPublikationen in der Regel das generische Maskulinum als geschlechtsneutrale Form verwendet. Diese Form impliziert immer alle Geschlechter. enverbrauch, Blutdruck und Blutzucker bis hin zu Herzfrequenz, Pulskurven und das EKG zu erfassen. So können Wearables auch dazu genutzt werden, einerseits symptomatische Arrhythmien aufzuzeichnen oder auf asymptomatische Rhythmusstörungen, wie z.B. Vorhofflimmern, zu screenen.

Wearables können daher als Supplement der klinischen Diagnostik von Arrhythmien eingesetzt werden, der Stellenwert von implantierbaren Rekordern (ILR) sowie deren Indikationsstellungen bleiben davon jedoch unbenommen.

Ziel dieses Positionspapiers ist es, einen Überblick zur aktuellen Datenlage und zum Einsatz der Wearables bei der Detektion von symptomatischen und asymptomatischen Arrhythmien zu geben. Zusätzlich werden gesundheitsökonomische und auch rechtliche Aspekte im Umgang mit Wearables beleuchtet.
Die frühe und korrekte Aufzeichnung und damit auch Diagnose von kardialen Arrhythmien haben wichtige therapeutische Implikationen. Ohne eine elektrokardiographische Dokumentation der Arrhythmie ist eine zielgerichtete Therapie nicht möglich. Da Arrhythmien teilweise nur sporadisch auftreten, stellt die Dokumentation im klinischen Alltag nicht selten eine große Herausforderung dar. Allein durch die Anamnese lassen sich die verschiedenen Differenzialdiagnosen supraventrikulärer oder ventrikulärer Rhythmusstörungen nicht sicher unterscheiden. Zur Aufzeichnung von Herzrhythmusstörungen stehen klassische Verfahren wie das 12-Kanal-EKG, das Langzeit-EKG über einen bis mehrere Tage und der externe oder implantierbare Ereignisrekorder zur Verfügung. Im Rahmen einer Beschwerdeabklärung liegt ein wichtiger Aspekt der Dokumentation von Herzrhythmusstörungen in

\section{Klinische Bedeutung der Arrhythmiedetektion}




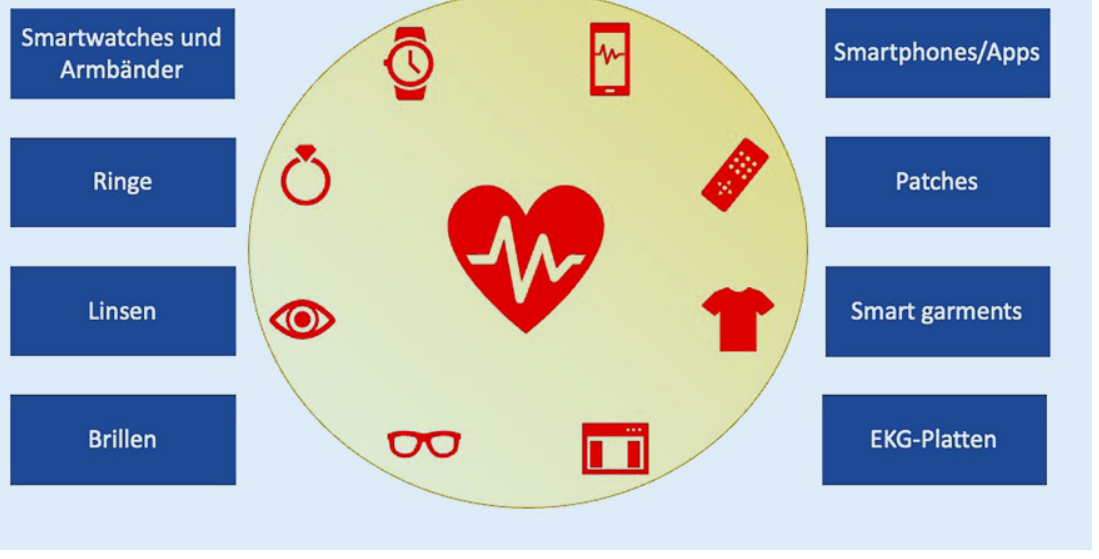

Abb. $1 \Delta$ Möglichkeiten der Arrhythmiedetektion mittels Wearables

der Abgrenzung organisch bedingter zu funktionellen oder psychosomatischen Beschwerden.

Die Erkennung dieser Rhythmusstörungen erfolgt aktuell meist im Kontext entsprechender Symptome. Herzrhythmusstörungen, wie v. a. das Vorhofflimmern, sind im Großteil der Fälle allerdings asymptomatisch. Eine gezielte Diagnostik wird dadurch erschwert. Vorhofflimmern stellt mit einer geschätzten Prävalenz von 1-2 \% in der Gesamtbevölkerung die häufigste Rhythmusstörung dar. Aufgrund der demografischen Entwicklung steigt die Prävalenz des Vorhofflimmerns in den nächsten Jahrzehnten weiter. Vorhofflimmern, ob asymptomatisch/subklinisch oder symptomatisch, ist mit einer signifikant erhöhten Morbidität und Mortalität assoziiert. Dazu zählen insbesondere Schlaganfälle, systemische Embolien und die Entstehung oder Aggravation einer Herzinsuffizienz. Patienten mit asymptomatischem Vorhofflimmern scheinen sogar eine schlechtere Prognose im Vergleich zu Patienten mit klassischer Vorhofflimmersymptomatik zu haben [57]. Infolge dessen zählt die Diagnose Vorhofflimmern laut statistischem Bundesamt seit Jahren zu den Top-5-Gründen für Krankenhauseinweisungen in Deutschland (www.destatis.de).

Aufgrund der hohen Prävalenz, der Morbiditäts- und Mortalitätssteigerung und der gesundheitsökonomischen Relevanz erscheint eine frühe Diagnose und Therapie von Vorhofflimmern sinnvoll.
Die aktuellen Guidelines der ESC und der EHRA empfehlen abhängig vom Alter bereits ein Screening auf subklinisches Vorhofflimmern. Bei Patienten ab 65 Jahren mit einer arteriellen Hypertonie ist ein opportunistisches Screening mit einer Klasse IB indiziert, bei Patienten ab 75 Jahren oder mit einem obstruktiven Schlafapnoesyndrom sollte ein systematisches Screening erwogen werden (Klasse IIa, B) [30].

\section{Screening- und Aufzeichnungs- strategien}

Die Möglichkeiten des Screenings auf subklinisches Vorhofflimmern, erstrecken sich aktuell vom täglichen Tasten des Pulses, wiederholten EKG-Registrierungen, der Aufzeichnung von LZ-EKGs bis hin zur Implantation von Loop-Rekordern. Auch Herzschrittmacher oder implantierbare Defibrillatoren können über die Detektion von sog. AHRE („atrial high rate episodes“) Hinweise auf asymptomatische atriale Tachyarrhythmien liefern. Wearables eröffnen eine neue Möglichkeit der Arrhythmiedetektion. Man unterscheidet prinzipiell 3 verschiedene Möglichkeiten, Arrhythmien mittels Wearables aufzuzeichnen.

Wearables können z.B. in Form eines Eventrekorders eingesetzt werden und erlauben die Dokumentation von symptomatischen Arrhythmien. Eventrekorder zur Detektion von Arrhythmien sind sinnvoll, sofern Arrhythmien seltener als 1-mal pro Woche auftreten. Der
Vorteil eines Wearable-basierten Eventrekorders ist, dass Patienten ihn jederzeit bei sich tragen und dieser im Falle einer Arrhythmie direkt verfügbar ist. Konventionelle externe Eventrekorder werden häufig nicht schnell genug aktiviert, um eine sporadische, nur kurz anhaltende Arrhythmie zu dokumentieren. Weitere Einsatzgebiete der Wearables sind die aktive und passive Arrhythmiedetektion zu Screeningzwecken. Unter aktiver Arrhythmiedetektion versteht man die Initiierung der Aufzeichnung durch den Patienten in vordefinierten Intervallen (1- bis 3-mal täglich). Durch diese Form der Arrhythmiedetektion wäre sowohl ein systematisches wie auch opportunistisches Screening auf Vorhofflimmern möglich. Des Weiteren erlaubt diese Form der Arrhythmiedetektion über festgelegte Aufzeichnungsintervalle die Bestimmung einer Arrhythmielast. Unter passivem Screening versteht man eine anwenderunabhängige Form der Arrhythmiedokumentation mittels kontinuierlicher oder periodischer automatischer Aktivierung der Aufzeichnung. Auch mittels dieses Verfahrens ist ein systematisches Screening auf Arrhythmien möglich. Eine Übersicht Wearable-basierter Arrhythmiedetektionsstrategien zeigt • Abb. 2.

\section{Übersicht über die technischen Optionen Wearable-basierter Arrhythmiedetektion}

Wearables in Form von Armbändern und Uhren zur Messung der Herzfrequenz haben sich innerhalb des letzten Jahrzehnts im Breitensport rasch verbreitet und werden dort vornehmlich zur Kontrolle der Herzfrequenz während des Trainings verwendet. Die technischen Möglichkeiten über die Messung der Herzfrequenz hinaus sind vielfältig und reichen von der Messung der Sauerstoffsättigung über die Bestimmung der Blutglukose bis hin zu EKGund Pulswellenaufzeichnungen [13]. In Verbindung mit entsprechenden Apps können diese Aufzeichnungen im Hinblick auf das Auftreten von Arrhythmien wie Vorhofflimmern analysiert werden.

Die Arrhythmiedetektion mittels Wearables erlangt aktuell besondere 
Hier steht eine Anzeige.

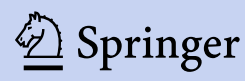


Aufmerksamkeit. Die Messung der Herzfrequenz, aber auch die Detektion von Herzrhythmusstörungen nutzt im Wesentlichen zwei unterschiedliche Technologien: die Photoplethysmographie (PPG) und die Elektrokardiographie (EKG) (• Tab. 1).

Die PPG basiert auf der Absorption von Infrarotstrahlung durch Hämoglobin. Aufgrund der unterschiedlichen Blutmenge während Systole und Diastole wird der Infrarotstrahl unterschiedlich stark absorbiert. Nimmt die Blutmenge während der Systole zu, wird die Absorption des Infrarotlichts größer, die Reflexion nimmt ab. Photoplethysmographiebasierte Wearables besitzen eine oder mehrere Lichtquellen sowie einen Photodetektor, der das vom Gewebe reflektierte Licht misst. Die Lichtquellen (Leuchtdioden) bestehen in den meisten Geräten aus Infrarotlicht emittierenden LEDs oder einer grünen LED-Diode. Photodioden messen die Intensität des vom $\mathrm{Ge}$ webe nicht absorbierten (reflektierten) Lichts. Über das Wearable wird ein Infrarotimpuls mit einer Wellenlänge von 800-1000 nm abgeben. Der reflektierte Anteil des Infrarotlichts wird vom Wearable gemessen und in eine Pulswellenkurve konvertiert. Über diese Pulswellenkurve wird die Pulsfrequenz gemessen. Zusätzlich können über spezielle Algorithmen die Regelmäßigkeit bzw. Unregelmäßigkeit der Pulswellenfolge analysiert und über die Pulsfrequenzvariabilität Rhythmusstörungen erkannt werden (• Abb. 3).

Photoplethysmographische Messungen erfolgen bei einem Großteil der Wearables am Handgelenk über Armbänder oder Uhren und ermöglichen dadurch ein aktives und passives Arrhythmiescreening. Über die SmartphoneKamera sind ebenfalls PPG-Aufzeichnungen möglich. Diese sind allerdings nur zum aktiven Arrhythmiescreening geeignet, um die Messung über die Positionierung auf einer Fingerkuppe oder über die Aufzeichnung des Gesichts zu ermöglichen.

Die EKG-basierte Arrhythmiedetektion mittels Wearables nutzt wie beim konventionellen EKG die Ableitung elektrischer Oberflächensignale, um ein 1- oder Mehrkanal-EKG abzuleiten (• Abb.4).

Kardiologe 2021 · 15:341-353 https://doi.org/10.1007/s12181-021-00488-3

(c) Deutsche Gesellschaft für Kardiologie - Herz- und Kreislaufforschung e.V. Published by Springer Medizin Verlag GmbH, ein Teil von Springer Nature - all rights reserved 2021

\section{Veltmann · J. R. Ehrlich · U. M. Gassner · B. Meder · M. Möckel · P. Radke · E. Scholz ·} H. Schneider $\cdot$ C. Stellbrink $\cdot$ D. Duncker

\section{Wearable-basierte Detektion von Arrhythmien. Positionspapier der Deutschen Gesellschaft für Kardiologie}

\section{Zusammenfassung}

Der technische Fortschritt in der Medizin hat dazu geführt, dass Herzfrequenz und Herzrhythmus nicht nur mittels professioneller Geräte aufgezeichnet werden können, sondern dies inzwischen auch vom Laien durch von jedermann erwerbbare sog. Wearables möglich ist. Unter Wearables versteht man direkt am Körper oder der Kleidung getragene elektronische Geräte, die in der Lage sind, biophysikalische Daten zu erheben. Zu den bekanntesten Wearables zählen Smartwatches, Armbänder und Brustgurte. Wearables sind in der Lage, Herzfrequenz, Pulskurven und das EKG zu erfassen. So können Wearables als Eventrekorder mit Symptom-getriggerter Aufzeichnung von Arrhythmien oder zum aktiven oder passiven Screening auf Vorhofflimmern genutzt werden. Dazu stehen prinzipiell 2 Verfahren zur Verfügung: die Elektrokardiographie und die Photoplethysmographie. Mit beiden Verfahren ist es inzwischen möglich, Software-unterstützt mit einer hohen Sensitivität und Spezifität Vorhofflimmern zu identifizieren. Ziel dieses Positionspapiers ist es, einen Überblick zur aktuellen Datenlage und zum Einsatz der Wearables bei der Detektion von symptomatischen und asymptomatischen Arrhythmien zu geben. Zusätzlich werden gesundheitsökonomische und auch rechtliche Aspekte im Umgang mit Wearables beleuchtet.

Schlüsselwörter

Wearables - Herzrhythmusstörungen . Vorhofflimmern $\cdot$ Screening $\cdot$ Smartwatch

\section{Wearable-based detection of arrhythmias. Position paper of the German Cardiac Society}

Abstract

The technical progress in medicine enables the acquisition of heart rate and heart rhythm not exclusively by professional medical devices but also for laypeople using wearables that can be purchased by everyone. Wearables are electronic devices worn directly on the body or clothing that are able to collect biophysical data. The most well-known wearables include smartwatches, bracelets and chest straps. Wearables are able to record heart rate, pulse curves and the ECG. They can be used as event recorders with symptom-triggered recording of arrhythmias or for active or passive screening for atrial fibrillation. There are basically two methods available: electrocardiography and photoplethysmography. With both methods it is possible to identify atrial fibrillation with a high level of sensitivity and specificity. The aim of this position paper is to provide an overview of the current data and the use of wearables in the detection of symptomatic and asymptomatic arrhythmias. In addition, health economic and legal aspects in dealing with wearables are addressed.

\section{Keywords}

Wearables · Arrhythmias · Atrial fibrillation . Screening $\cdot$ Smartwatch
Mit entsprechenden Applikationen können dann die EKG analysiert und Diagnosen gestellt werden. Die ersten Wearables mit EKG-Funktion zeichnen ein Oberflächen-EKG mithilfe von zusätzlicher Hardware in Form von „externen“, nicht in das Wearable integrierten Elektroden auf. Diese sind via Kabel oder auch kabellos mit einem Smartphone oder Tablet verbunden. Die generierten EKG werden vom Weara- ble auf das Smartphone oder Tablet übertragen, dort gespeichert und mit entsprechenden Applikationen analysiert und bewertet. Zudem können die gespeicherten EKG via E-Mail oder Messengerdienst versandt werden. Die EKGAbleitung erfolgt durch klassische Kabelbäume mit auf der Haut zu fixierenden Elektroden bis hin zu „Hand“- oder „Daumen“-EKG-Geräten. Ein als Medizinprodukt zertifiziertes System ist in 


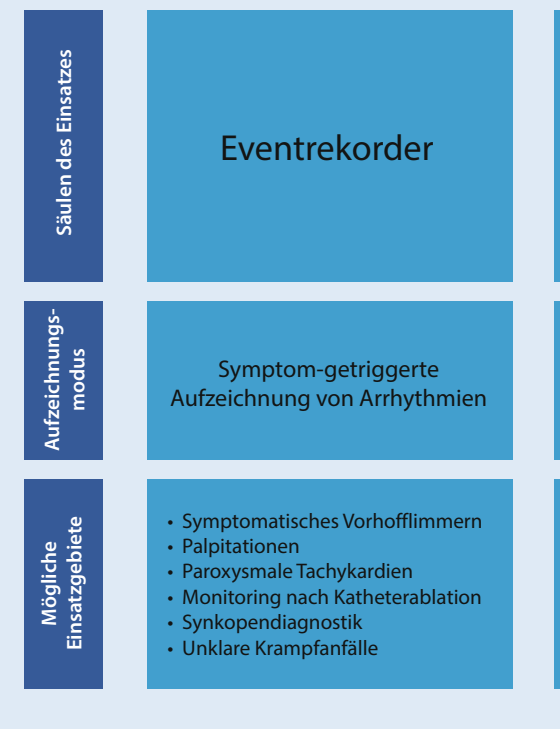

der Lage, über 4 Oberflächenelektroden ein 12- bzw. 22-Kanal-EKG zu generieren. Der Nutzen dieser vereinfachten EKG-Ableitung im klinischen Einsatz konnte kürzlich gezeigt werden [59]. Andere Systeme nutzen beispielsweise adhäsive Patches, Brustbänder, Armbänder oder auch Hemden mit integrierten Elektroden [9, 17, 19, 28, 43].

Weiter verbreitet sind die Hand- oder Daumen-EKGs. Diese Geräte besitzen 2 nahe beieinanderliegende Elektroden in Form von Metallplatten, auf die jeweils ein Finger oder Daumen beider Hände gelegt wird, oder auch einen Stab, der an den Kontaktstellen mit der rechten und linken Hand umgriffen wird. So wird ein 1-Kanal-EKG registriert, das der Ableitung I nach Einthoven entspricht (• Abb. 4b; [23, 26]).

Mittlerweise sind auch Smartwatches erhältlich, die ein 1-Kanal-EKG ohne zusätzliche Hardware oder weitere externe Elektroden ableiten können. Diese Wearables haben den großen Vorteil der leichteren Handhabung und schnelleren Verfügbarkeit, da nicht mehrere Devices zur EKG-Dokumentation vorgehalten werden müssen. Die Ableitung eines 1-Kanal-EKGs, der Ableitung I nach Einthoven entsprechend, erfolgt einerseits über den Kontakt an der Rückseite des Uhrgehäuses mit der Haut des linken Handgelenks (Pluspol). Den Minuspol, der mit einem Finger der kontralateralen Hand berührt wird, stellt eine Elektrode

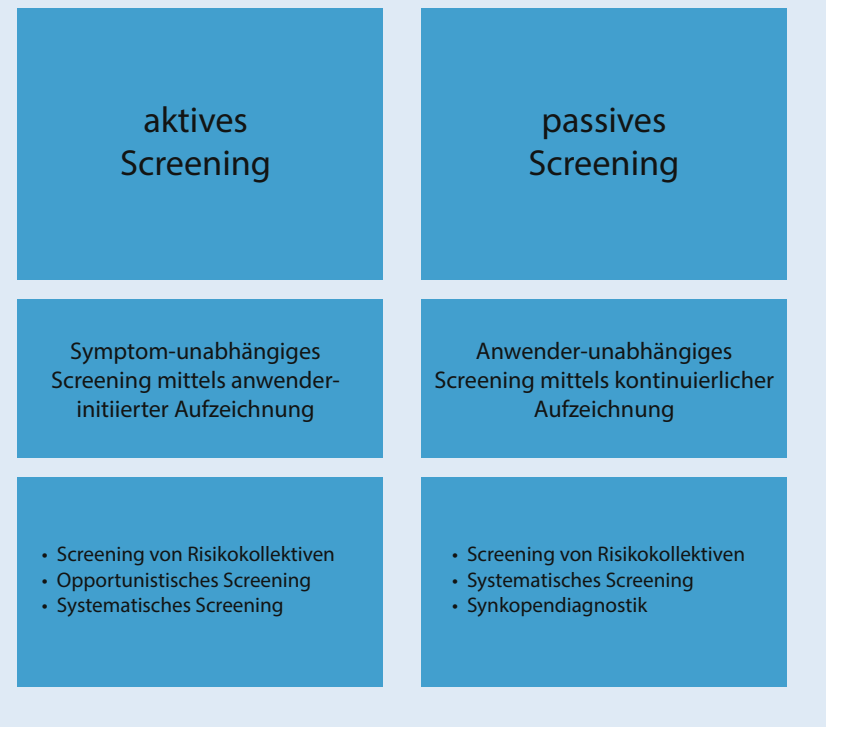

Abb. $2<$ Einsatzmöglichkeiten von Wearables zur Arrhythmiedetektion im Armband der Uhr, die Krone oder das Gehäuse der Smartwatch, dar (• Abb. 5). Über Veränderungen der Position der Uhr und Änderung der Polaritäten ist es sogar möglich, alle Ableitungen nach Einthoven und einige Brustwandableitungen nach Wilson durch sukzessive 1-Kanal-Ableitungen aufzuzeichnen (• Abb. 6; [4, 54]). Über die Modifikation der Ableitungen erscheint selbst die Beurteilung der ST-Strecke bei Verdacht auf Myokardischämie möglich [42].

\section{Übersicht und Interpretation der klinischen Daten der verschiedenen Methoden}

Die Genauigkeit von Wearables hinsichtlich Detektion und Differenzierung von Arrhythmien hängt einerseits von der verwendeten Messtechnik und andererseits von der zu erkennenden Arrhythmie ab.

Ein Großteil der eingesetzten Geräte ermittelt die Herzfrequenz mithilfe optischer Sensoren (PPG). Vorteil dieser Technik ist, dass sie kostengünstig und technisch leicht integrierbar ist. Zudem kommt sie ohne Elektroden aus. Häufig in Wearables am Handgelenk eingesetzt, eignet sich die PPG zur Erfassung der Herzfrequenz in Ruhe und unter Belastung. Im direkten Vergleich zum EKG zeigt sich abhängig vom eingesetzten System hier eine akzeptable Korrelation, wobei die Präzision bei höherer Herzfre- quenz sowie beim Vorliegen von Vorhofflimmern abnimmt $[34,66]$. Auch die Art der körperlichen Belastung und damit verbundene Bewegung der jeweiligen Extremität, an der die Messung stattfindet, kann die korrekte Ermittlung der Herzfrequenz beeinflussen [21]. Neben der alleinigen Bestimmung der Frequenz kann die PPG mit akzeptablen Erkennungsraten auch zur Detektion von Bradykardien, Tachykardien, Asystolien, ventrikulären Tachykardien und Kammerflimmern eingesetzt werden [47]. In kleineren experimentellen Studien konnte auch die Detektion von Extrasystolen gezeigt werden, wobei mithilfe differenzierter Analyseverfahren der Pulswellenkurve sogar eine Differenzierung zwischen supraventrikulärem und ventrikulärem Ursprung möglich zu sein scheint $[50,58]$.

Zahlreiche Studien haben sich mit dem Einsatz der PPG zur Detektion von Vorhofflimmern befasst. Obwohl es sich hierbei nicht um Wearables im engeren Sinne handelt, sind hier als Pionierleistung der Einsatz der Kamera eines iPhone sowie Videoaufnahmen des Gesichts als Datenquelle zur Vorhofflimmerdetektion zu nennen [10, 41, 67, 68]. Ebenfalls mithilfe einer Smartphone-Applikation konnten im Rahmen einer klinischen Studie zum Screening nach Vorhofflimmern eine hohe Messgenauigkeit und gute Übereinstimmung mit EKG-basierten Methoden gezeigt werden [8]. Eingesetzt in klassischen 
Tab. 1 Unterschiede zwischen Photoplethysmographie- und Elektrokardiogramm-basierter Arrhythmiedetektion durch Wearables

\begin{tabular}{|c|c|c|}
\hline & Photoplethysmographie & Elektrokardiographie \\
\hline Technologie & Pulswellenanalyse & Herzstromkurve \\
\hline Voraussetzungen & $\begin{array}{l}\text { Wearable, Smartphone- } \\
\text { Kamera, Applikation }\end{array}$ & Wearable, Elektrode(n), Applikation \\
\hline Messmodus & $\begin{array}{l}\text { Kontinuierlich, opportunis- } \\
\text { tisch, systematisch }\end{array}$ & $\begin{array}{l}\text { (Selten kontinuierlich), opportunistisch, } \\
\text { systematisch }\end{array}$ \\
\hline Screeningmöglichkeit & $\begin{array}{l}\text { Eventrekorder, pas- } \\
\text { siv > aktiv }\end{array}$ & Eventrekorder, aktiv > passiv \\
\hline Messgrößen & $\begin{array}{l}\text { Herzfrequenz, Herzfre- } \\
\text { quenzvariabilität }\end{array}$ & $\begin{array}{l}\text { Herzfrequenz, Herzfrequenzvariabilität, } \\
\text { Extrasystolen, komplette EKG-Analyse }\end{array}$ \\
\hline Analysedauer & Ca. $30-60 \mathrm{~s}$ & Ca. $30-60 \mathrm{~s}$ \\
\hline $\begin{array}{l}\text { Sensitivität Vorhof- } \\
\text { flimmerdiagnose [30] }\end{array}$ & $91,5-98,5 \%$ & $94-98 \%$ \\
\hline $\begin{array}{l}\text { Spezifität Vorhof- } \\
\text { flimmerdiagnose [30] }\end{array}$ & $91,4-100 \%$ & $76-95 \%$ \\
\hline Artefakte & $\begin{array}{l}\text { Bewegungs- und Kopp- } \\
\text { lungsartefakte am Sensor }\end{array}$ & $\begin{array}{l}\text { Bewegungs- und Kopplungsartefakte an } \\
\text { den Elektroden }\end{array}$ \\
\hline
\end{tabular}

Wearables am Handgelenk, zeigen PPGbasierte Analysemethoden eine Sensitivität und Spezifität von deutlich über $90 \%$ bei der Erkennung von Vorhofflimmern [5, 16, 44, 62]. Die Detektion erfolgte in diesem Fall mithilfe eines Algorithmus, basierend auf trainierter künstlicher Intelligenz [62]. Die Algorithmen zur Detektion von Vorhofflimmern basieren auf der Unregelmäßigkeit der R-RIntervalle, wie sie bereits seit Jahren bei implantierbaren Looprekordern zum Einsatz kommen [55].

Sogar eine Differenzierung zwischen Vorhofflimmern und Vorhofflattern ist mittels Photoplethysmographie beschrieben [14]. Häufig werden jedoch beispielsweise in groß angelegten Screeningstudien wie der Apple Heart Study diese beiden Arrhythmieformen unter dem gemeinsamen Begriff „Vorhofflimmern“ subsumiert [63]. Obwohl eine Differenzierung zwischen Vorhofflattern und Vorhofflimmern über die zeitliche Abfolge der R-R-Intervalle prinzipiell mit hoher Sensitivität und Spezifität möglich ist, ist für die exakte Interpretation die Analyse der atrialen Aktivierung erforderlich, was nur elektrokardiographisch, nicht aber mittels PPG möglich ist [56].

Neben der PPG findet auch die klassische Ableitung von Elektrogrammen Einsatz in Wearables. Dabei erfolgt eine direkte EKG-Ableitung über die Thoraxwand oder die Extremitäten, die neben einer Beurteilung der atrialen Aktivität auch die EKG-morphologischer Charakteristika erlaubt. Im Gegensatz zur PPG ist die Messung von Elektrokardiogrammen als Goldstandard in der Diagnostik von Arrhythmien anzusehen. Dieser Goldstandard gilt jedoch bislang für eine ärztliche Befundung der EKG-Registrierung, bei der die Expertise und die Befundungshoheit in ärztlicher Hand liegen. Zwischenzeitlich wurden Algorithmen entwickelt, die eine automatisierte, Arzt-unabhängige Interpretation zur Verfügung zu stellen. Diese sind in der Lage, Artefakte zu eliminieren, QRSKomplexe zu erkennen und eine automatische Segmentierung vorzunehmen [15]. Die Signalqualität von EKG-basierten Wearables ist dabei vergleichbar mit der Signalqualität von klassischen EKGs oder Langzeit-EKGs [25, 45, 60]. Auf diese Weise sind differenzierte Messungen, wie beispielsweise die Dauer des QTIntervalls, möglich [7]. Eine Übersicht über aktuell verfügbare Systeme bietet die Übersichtsarbeit von Bansal und Joshi [2]. Im Bereich der 1-Kanal-EKGGeräte konnte in den meisten Studien eine sehr hohe Sensitivität und Spezifität $(>90 \%)$ für die Detektion von Vorhofflimmern gezeigt werden [32, 33, 35, 38]. Für Mehr-Kanal-Geräte konnte in puncto Arrhythmieerkennung sogar eine Überlegenheit gegenüber herkömmlichen Langzeit-EKG-Geräten nachgewiesen werden $[3,53]$.
Im Vergleich zum selbstständigen Screenen auf Vorhofflimmern durch Tasten des Pulses zeigen sowohl PPG als auch EKG-basierte Techniken deutlich höhere Erkennungsraten. In einer prospektiv angelegten Studie an einem älteren Patientenkollektiv konnte durch Palpation des Pulses eine Erkennungsrate von neu aufgetretenem Vorhofflimmern von $70 \%$ erreicht werden [31]. Die Erkennungsraten verschiedener Techniken und Geräte im Vergleich zum 12-KanalEKG als Goldstandard wurden in einer Metaanalyse zusammengefasst [61]. Die besten Ergebnisse lieferten dabei EKGGeräte mit reduzierter Elektrodenzahl sowie Blutdruckmessgeräte. Vergleichbar gute und zum Teil bessere Resultate konnten durch Smartphone-Applikationen gezeigt werden. Die Erkennung von Vorhofflimmern durch Palpation des Pulses war hingegen allen oben genannten Techniken unterlegen [61].

\section{Wearable-basierte Arrhythmie- detektion in verschiedenen Populationen}

Die aktuellen Leitlinien der ESC empfehlen in verschiedenen Risikopopulationen ein opportunistisches oder systematisches Vorhofflimmerscreening [30]. Ziel einer frühzeitigen Diagnose von subklinischem Vorhofflimmern und in Abhängigkeit des Schlaganfallrisikos der Beginn einer oralen Antikoagulation oder auch Einleitung einer rhythmuskontrollierenden Therapie ist die Reduktion der vorhofflimmerbedingten Morbidität und Mortalität. Dadurch wäre zudem eine Reduktion der Kosten im Gesundheitssystem zu erwarten [36].

Screening auf Vorhofflimmern sollte möglichst kontinuierlich erfolgen. Aktuelle Strategien bestehen in der Durchführung von Langzeit-EKG-, 12-KanalEKG- oder auch Eventrekorderaufzeichnungen $\mathrm{zu}$ verschiedenen Zeiten und Intervallen. Allerdings sind diese Verfahren nicht in der Lage, subklinisches Vorhofflimmern suffizient zu detektieren. Diederichsen und Kollegen haben verschiedene Screeningstrategien, basierend auf 12-Kanal-EKG, klassischem 24bis $72-\mathrm{h}-\mathrm{LZ}-\mathrm{EKG}$ bis hin zum LZ-EKG über 30 Tage, in verschiedenen Interval- 


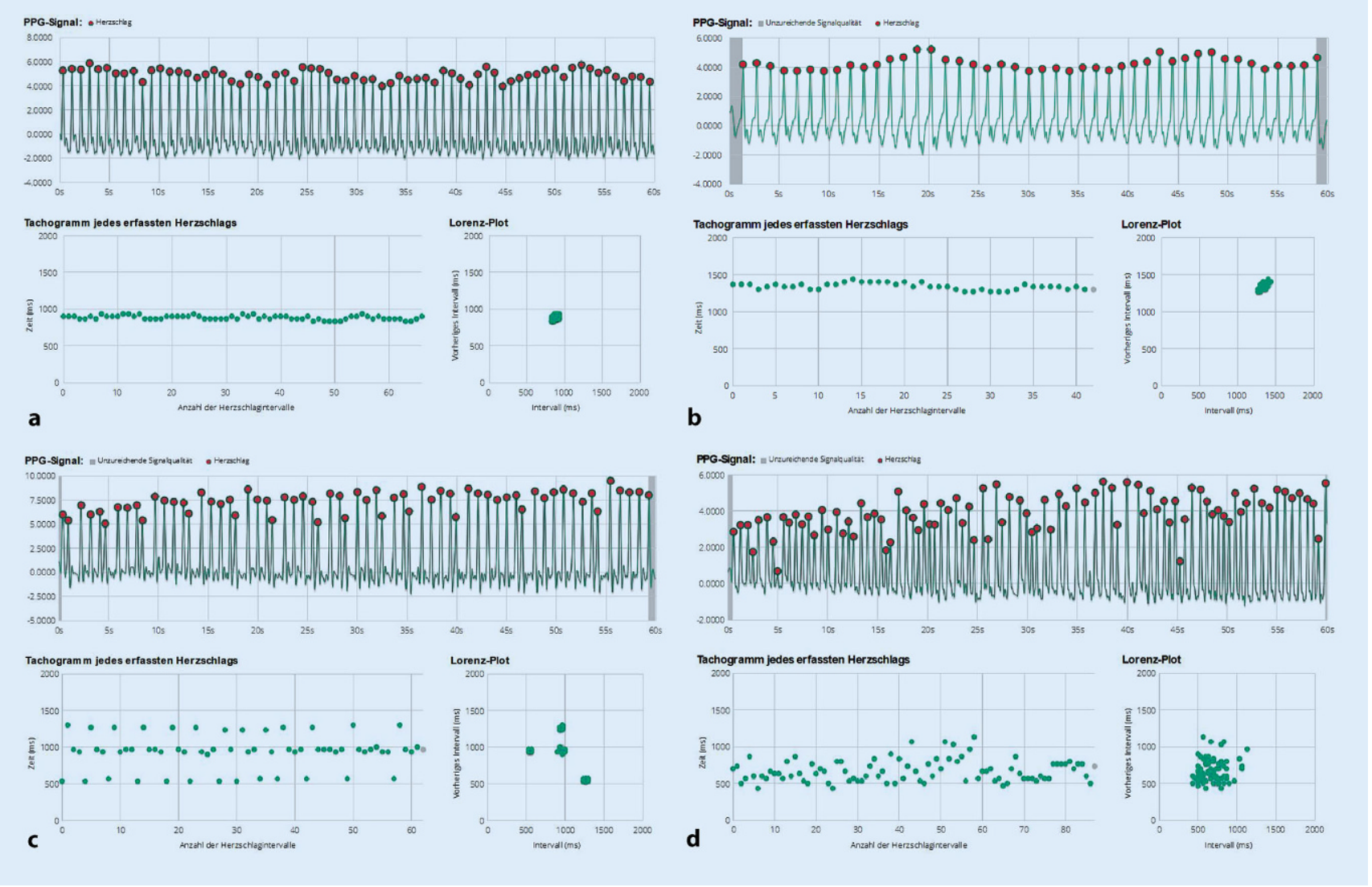

Abb. 3 ॥ Photoplethysmographische Aufzeichnung (PPG-Signal, Tachogramm und Lorenz-Plot). a Aufzeichnung mit Smartphone-Kamera, Sinusrhythmus. b Aufzeichnung mit Armband, Sinusrhythmus. c Aufzeichnung mit Smartphone-Kamera, ventrikuläre Extrasystolen. d Aufzeichnung mit Smartphone-Kamera, Vorhofflimmern

len simuliert und mit dem Goldstandard des implantierten Looprekorders verglichen. Mittels kontinuierlichen Looprekorder-Monitorings konnten bei 590 Patienten insgesamt 20.110 Vorhofflimmerepisoden $>6$ min diagnostiziert werden. Ein über 2 Jahre monatlich durchgeführtes 24-h-LZ-EKG hatte in dieser Studie eine Sensitivität von nur $30 \%$, Vorhofflimmern zu detektieren. Die höchste Sensitivität (55\%), Vorhofflimmern neu zu diagnostizieren, hatte ein über 3 Jahre jährlich durchgeführtes 30-Tage-LZ-EKG. Die Autoren konnten zusätzlich zeigen, dass die Sensitivität steigt, wenn die gleiche Screeningdauer auf mehrere Zeiträume aufgeteilt wird. Ein 3-maliges Screening über $24 \mathrm{~h}$ zeigte eine höhere Sensitivität als 1-malig über $72 \mathrm{~h} \mathrm{[11].} \mathrm{Quer} \mathrm{et} \mathrm{al.} \mathrm{bestätigten,} \mathrm{dass}$ mit einem 2-mal täglich durchgeführten EKG im Vergleich zum kontinuierlichen Monitoring nur ca. $50 \%$ der Vorhofflimmerepisoden aufgezeichnet wer- den konnten [52]. In der REHEARSEAF-Studie konnte gezeigt werden, dass ein 2-mal wöchentlich aufgezeichnetes Wearable-EKG hinsichtlich der Detektion von Vorhofflimmern gegenüber der Standardversorgung überlegen ist. Mittels Wearables konnten bei $3,8 \%$ des Studienkollektives von Menschen über 65 Vorhofflimmern neu diagnostiziert werden. Im Kontrollarm lag die Rate von neu diagnostiziertem Vorhofflimmern nur bei $1,0 \%$ [26].

Wearables sind potenziell in der Lage die diagnostische Lücke zu schließen, die das konventionelle EKG-basierte Screening hinterlässt [18]. Sie ermöglichen ein aktives oder passives Screening über EKG- oder PPG-basierte Verfahren.

Diverse Screeningmethoden wurden an unterschiedlichen Populationen untersucht. So wurde schon vor Jahren mittels Wearable-EKG die Prävalenz von Vorhofflimmern im Rahmen einer Grippeimpfung oder nach öffentlichem
Aufruf in der Allgemeinbevölkerung untersucht $[8,46]$. In diesen Studien konnte Vorhofflimmern mit einer in dem jeweiligen Studienkollektiv zu erwartenden Prävalenz diagnostiziert werden. Auch während Apothekenbesuchen ist das Screening auf Vorhofflimmern möglich $[69,70]$. In einer kürzlich veröffentlichten deutschen Studie wurde das während des Apothekenbesuchs diagnostizierte, bis dahin unbekannte Vorhofflimmern als negativer Prädiktor für das 1-Jahres-Überleben identifiziert [70]. Das im Rahmen der ESC-Leitlinie empfohlene opportunistische Screening lässt sich auch im hausärztlichen Umfeld umsetzen $[1,22]$. Bei jedem Hausarztbesuch wurde ein 30-sekündiges Wearable-EKG aufgezeichnet und konnte in dem Studienkollektiv >65 Jahre bei $6,2-7,0 \%$ Vorhofflimmern neu diagnostizieren. In über der Hälfe der Patienten resultierte aus der Vorhofflimmerdiagnose der 


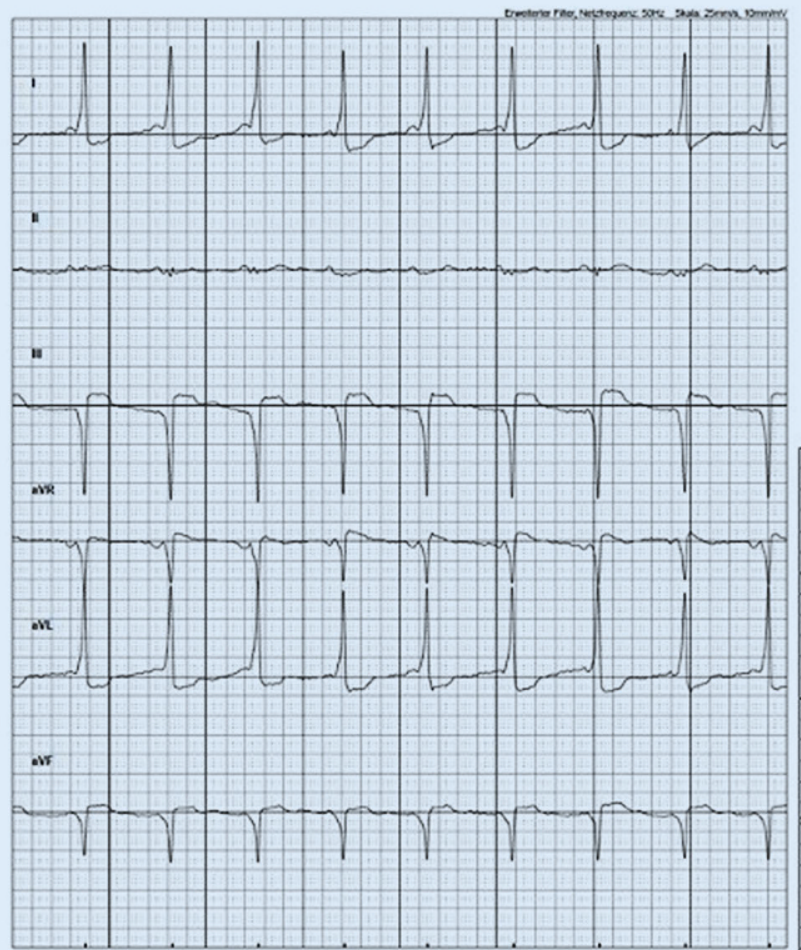

a

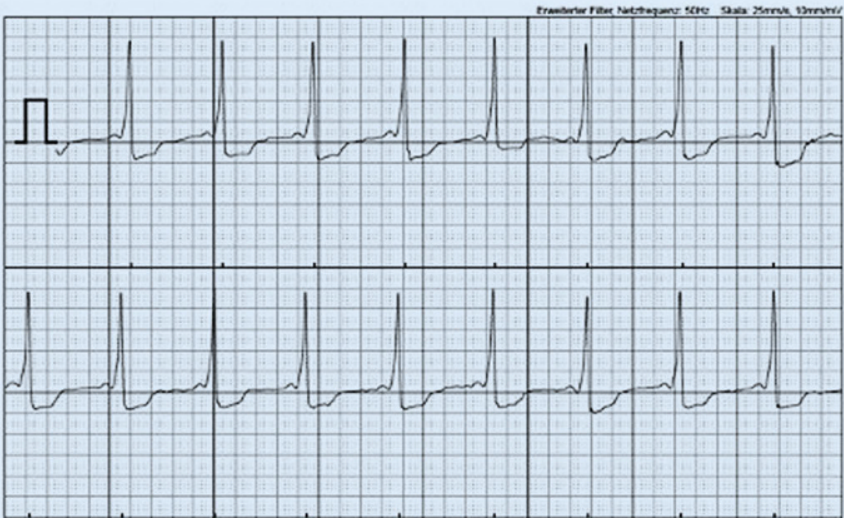

b

Abb. 4 \ EKG-Aufzeichnung eines Patienten mit Präexzitation mit einem a 6-Kanal-EKG (KardiaMobile 6L) und b einem 1-Kanal-EKG (Kardia, beides Alivecor)

Beginn einer oralen Antikoagulation [22].

Mittels PPG wurde auch in der Allgemeinbevölkerung auf Vorhofflimmern untersucht. In einer Studie aus Belgien wurden Probanden in der Tageszeitung aufgerufen, sich eine App herunterzuladen und über die Smartphone-Kamera auf Vorhofflimmern zu screenen. Sieben Tage lang sollten die Studienteilnehmer 2-mal täglich eine PPG-Aufzeichnung durchführen. Insgesamt nahmen 12.328 Personen an der Studie teil und übermittelten 120.446 PPG. Auf Vorhofflimmern hinweisende PPG wurde in 1,1 \% der Fälle dokumentiert. Personen mit vorhofflimmerverdächtigem PPG waren signifikant älter und Männer mit einem höheren BMI verglichen mit Personen mit normalen PPG [65].

Auch in speziellen Kollektiven wurden Wearables zum Screening auf Vorhofflimmern eingesetzt. Nach herzchirurgischer Operation oder Katheterablation ist der Einsatz von Wearables zur Detektion von postoperativem oder postinterven- tionellem Vorhofflimmern denkbar. In einer kleinen Studie konnte bei Patienten mit postoperativem Vorhofflimmern bei $24 \%$ der Patienten nach Krankenhausentlassung die Rhythmusstörung erneut mittels eines Wearables diagnostiziert werden; $80 \%$ der Patienten mit Vorhofflimmerdiagnose hatten eine Indikation für eine orale Antikoagulation [37].

Mehrere Studien haben das passive Vorhofflimmerscreening mittels PPG über eine Smartwatch oder ein Armband untersucht. Als größte Studien sind die Apple Heart-Studie und die MAFA II-Studie zu nennen [24, 48]. In beiden Studien wurde die Allgemeinbevölkerung aufgerufen an diesen PPGbasierten Screeningstudien teilzunehmen. Es nahmen initial über 400.000 bzw. 200.000 Personen teil. Die Prävalenz vorhofflimmerverdächtiger PPG lag bei 0,52 und $0,2 \%$. Durch gezielte weiterführende Diagnostik wurde im Anschluss bei $87 \%$ in der MAFA IIStudie und $84 \%$ in der Apple HeartStudie Vorhofflimmern diagnostiziert.
Einschränkend ist zu sagen, dass die Patientenkollektive aus überwiegend jungen Patienten bestanden, bei denen die Vorhofflimmerprävalenz sehr niedrig ist. Das relevantere Kollektiv $>65$ Jahre war deutlich unterrepräsentiert. Des Weiteren hat der Großteil der Patienten nach vorhofflimmerverdächtigem PPG die empfohlene weiterführende Diagnostik zur Bestätigung der Vorhofflimmerdiagnose nicht in Anspruch genommen.

Eine definitive Vorhofflimmerdiagnose via PPG ist derzeit nicht möglich. Von daher ist bei Vorhofflimmerverdacht, basierend auf einem PPG, eine elektrokardiographische Bestätigung anzustreben, bevor eine therapeutische Konsequenz gezogen werden kann. Allerdings zeigen die auf der Herzfrequenzvariabilität basierenden Algorithmen ähnlich gute Sensitivitäten und Spezifitäten von EKG und PPG [6]. Inwieweit eine Vorhofflimmerdiagnose, basierend auf einem PPG, in Zukunft möglich sein wird, müssen weitere Studien zeigen. Die aktuell verfügbaren Wearables in Kombination mit 


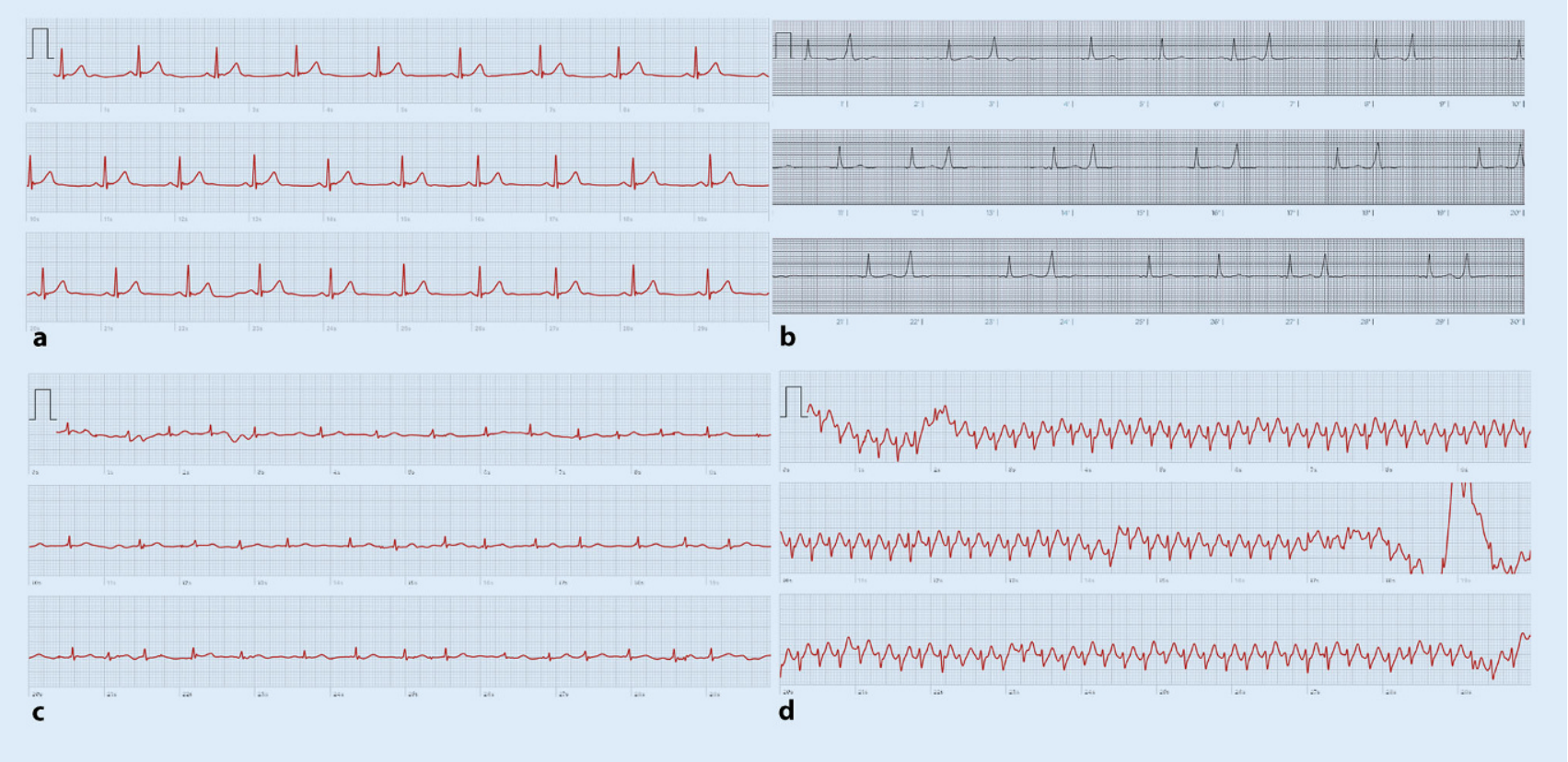

Abb. 5 \ EKG-Aufzeichnungen mit Smartwatches. a Sinusrhythmus, Apple Watch (Apple, Cupertino, CA, USA). b Sinusrhythmus mit ventrikulären Extrasystolen, ScanWatch (Withings, Issy-les-Moulineaux, Frankreich). c Vorhofflimmern, Apple Watch. d Regelmäßige Schmalkomplextachykardie, Apple Watch

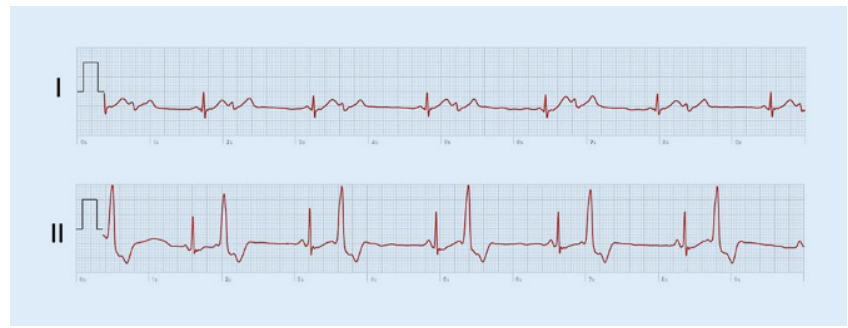

Abb. $6 \Delta$ Aufzeichnung eines ventrikulären Bigeminus mit einer Apple Watch: Ableitung I (linkes Handgelenk - rechter Zeigefinger), Ableitung II (linker Unterschenkel - rechter Zeigefinger)

entsprechenden Applikationen und Algorithmen zeigen eine hohe Übereinstimmung mit der klassischen EKG-Aufzeichnung. Die aktuelle Datenlage zeigt, dass Wearables prinzipiell zum Vorhofflimmerscreening eingesetzt werden können und von Patienten als auch von den behandelnden Ärzten angenommen werden [22].

Outcomestudien zum Wearable-basierten Vorhofflimmerscreening existieren aktuell noch nicht, sodass der Nutzen über die Diagnose hinaus noch nicht gezeigt werden konnte. Studien in speziellen Kollektiven sind auf dem Weg (NCT04282993, Apple Heartline NCT04276441). Letztlich erscheint der Einsatz der Wearables zunächst in den Risikokollektiven sinnvoll zu sein, wenn sich eine unmittelbare therapeutische
Konsequenz aus der Vorhofflimmerdiagnose in Form einer oralen Antikoagulation oder Rhythmuskontrolle ergibt.

\section{Möglicher Einsatz im klinischen Alltag und Umgang mit Wearable-basiertem „Vorhofflimmern"}

\section{Herausforderungen hinsichtlich weiterführender diagnostischer Maßnahmen}

Der Markt bietet eine Vielzahl von Wearables, die von jedermann käuflich erworben werden können. Viele dieser Geräte sind nicht als Medizinprodukt zertifiziert und werden als Lifestyleprodukt eingestuft und beworben. Daher werden Ärzte in Zukunft zunehmend mit Auf- zeichnungen und Daten von Wearables konfrontiert werden. Die Daten und Aufzeichnungen nicht zertifizierter Geräte sollten schon aus Haftungsgründen nicht für diagnostische oder gar therapeutische Zwecke genutzt werden. Einige Wearables sind allerdings als Medizinprodukt zertifiziert und können und sollten daher akzeptiert und genutzt werden.

Die aktuelle Datenlage erlaubt, Wearables einerseits als Eventrekorder oder zum Screening auf Vorhofflimmern zu nutzen. Bei Smartphone-unerfahrenen Patienten ist die Nutzung von Wearables $\mathrm{zu}$ diagnostischen Zwecken sicherlich eingeschränkt. Gerade bei älteren $\mathrm{Pa}$ tientenkollektiven besteht noch keine ausreichende Erfahrung im sicheren Umgang mit Smartphones und applikationsbasierten Diagnostika wie der PPG mittels Smartphone-Kamera [51].

Zusätzlich eingeschränkt wird die Nutzung von Wearables durch die entstehenden Kosten. Wearables und Applikationen sind kostenpflichtig und teilweise sehr kostenintensiv, sodass sich nicht jeder Patient diese Form der Diagnostik leisten kann. Diese Kostenproblematik hat sich indes inzwischen teilweise entschärft. Gesetzlich Krankenversicherte haben seit 19.12.2019 
grundsätzlich einen Anspruch auf digitale Gesundheitsanwendungen (DiGA). Bei DiGA handelt es sich - vereinfacht gesagt - um Medizinprodukte der Klasse I oder IIa, die hauptsächlich auf digitalen Technologien beruhen und bei der Untersuchung bzw. Behandlung von Krankheiten unterstützen. Der Versorgungsanspruch bezieht sich auf solche Produkte, die vom Bundesinstitut für Arzneimittel und Medizinprodukte (BfArM) auf Antrag des Herstellers in das DiGA-Verzeichnis aufgenommen wurden. Die Versicherten können diese Ansprüche auf doppelte Weise realisieren: entweder über den Weg einer Verordnung durch den behandelnden Arzt (in der Praxis der Regelfall) oder auch durch Genehmigung der Krankenkasse, wenn die Indikation, für die die DiGA bestimmt ist, durch einen Arzt bestätigt wird. Sowohl der behandelnde Arzt als auch die Krankenkasse können jeweils eigenständig und unabhängig voneinander darüber entscheiden, ob im Einzelfall eine vollständige oder teilweise Kostenübernahme erfolgt. Allerdings wurden bis März 2021 noch keine DiGA für Arrhythmiediagnostik in das DiGAVerzeichnis aufgenommen.

Im Falle einer EKG-basierten Dokumentation einer Rhythmusstörung kann diese zu weiterführender Diagnostik und Therapie eingesetzt werden. Auch die aktuellen Leitlinien zu Vorhofflimmern der ESC sehen eine Dokumentation von Vorhofflimmern über eine Aufzeichnung von $30 \mathrm{~s}$ mittels 1-Kanal-EKG als diagnostisch an. Im Falle einer PPG-basierten Dokumentation einer Arrhythmie ist vor Indikationsstellung einer invasiven Diagnostik oder Therapie eine elektrokardiographische Aufzeichnung der Arrhythmie anzustreben, bevor eine definitive Diagnose oder gar weiterführende Therapie eingeleitet wird.

Beim passiven Vorhofflimmerscreening mittels PPG ist ebenfalls eine elektrokardiographische Bestätigung erforderlich. Diese ist Voraussetzung für eine therapeutische Konsequenz im Sinne einer oralen Antikoagulation oder auch rhythmuskontrollierenden Therapie.

Patienten mit Vorhofflimmern stellen eine extrem heterogene Gruppe von $\mathrm{Pa}$ tienten dar, deren Morbidität ein weites
Spektrum aufweist. Bisher liegen keine Daten vor, welche Patientengruppen bevorzugt Wearables aus eigenem Antrieb benutzen oder umgekehrt welchen Gruppen diese aktiv empfohlen werden könnten.

Effektiv zeigen jedoch verschiedene kürzlich publizierte Erhebungen der EHRA [39, 40] und der HRS [12], dass die medizinischen Informationen aus Wearables zur Rhythmusdokumentation in der klinischen Praxis bereits eingesetzt werden, und dokumentieren ein grundsätzliches Vertrauen in die diagnostische Qualität dieser Devices. Bedenken bestehen v. a. bezüglich der zu bewältigenden Datenmenge [39] sowie der Datenintegration in die (elektronische) Patientenakte [12]. Eine eindeutige Patientenzuordnung der getätigten Rhythmusaufzeichnungen muss dabei gewährleistet sein, insbesondere bei therapierelevanten Befunden.

\section{Resultierende therapeutische Konsequenzen}

Eine valide, Wearable-basierte Plattform muss für Ärzte und Nutzer absolut zuverlässig sein in Bezug auf eine schnelle und einfache Bedienung, verlässliche Technik, sichere rechtliche Rahmenbedingungen und kurze Reaktionszeiten. Bereits jetzt gibt es Plattformen, die beispielsweise eine EKG-Analyse und eine digitale Gesundheitsakte für niedergelassene Kardiologen und deren Patienten zur Verfügung stellen und auch eine 24-h/7-d-Bereitschaft zur Reaktion auf EKG-Aufzeichnungen vorhalten. Diese Infrastruktur kann bereits jetzt genutzt werden und sollte in Zukunft unter kardiologischem Einfluss weiter ausgebaut werden. Hier spielt die rechtliche Sicherheit des Arztes (Fernbehandlung, haftungsrechtliche Fragen) und des Patienten (Datensicherheit, Verlässlichkeit) eine zentrale Rolle. Aber auch die Vergütung der Strukturen sowie der ärztlichen Leistung muss hier sichergestellt sein.

Telemedizinische Behandlungskonzepte finden sich inzwischen in zahlreichen Bereichen der ambulanten Medizin. Aufgrund der faktischen telemedizinischen Behandlungssituation ist eine Option, zukünftig vorab mit den Patien- ten über die Grenzen und Möglichkeiten der Nutzung persönlicher Wearables zu sprechen. Darüber hinaus sollte geklärt werden, wie die ärztliche Leistung in diesem Kontext budgetär zu bewerten ist. Hier fehlen bisher eindeutige rechtliche Rahmenbedingungen.

Viele Wearables sind bereits als Medizinprodukt zertifiziert. In diesem Falle ist rechtlich verbindlich entsprechend der Zweckbestimmung des Herstellers festgelegt, welche Informationen des Wearables medizinisch verwertet werden dürfen (z.B. Herzfrequenz, Rhythmus, andere Parameter). Bisher fehlen noch Standards für die telemedizinische Übermittlung dieser Daten und Integration in die Patientenakte, z. B. der EKGRegistrierung. Der Entwurf eines Gesetzes zur digitalen Modernisierung von Versorgung und Pflege (Digitale-Versorgung-und-Pflege-ModernisierungsGesetz - DVPMG) sieht vor, dass Daten des Versicherten bei entsprechender Einwilligung aus der DiGA von ihrem Hersteller über den Anbieter der elektronischen Patientenakte (ePA) über eine Schnittstelle in diese übermittelt werden.

Somit kann der Patient im Rahmen einer persönlichen Vorstellung darüber beraten werden, dass ein Wearable zur Dokumentation und Interpretation einer Arrhythmieepisode hilfreich sein kann.

Im Zuge der Kontaktrestriktionen während der COVID-Pandemie wurden die Telemedizin und digitale Medizinkonzepte stark angefeuert [64] Hier konnte im Rahmen des TeleCheck-AFProjektes europaweit ein Arzt-initiiertes, aber Patienten-zentriertes Rhythmusmonitoring mittels PPG-App etabliert werden [20, 29, 49]. Der Einsatz von Wearables in der klinischen Routine wurde durch die Pandemie deutlich verstärkt [27].

\section{Rechtlicher Rahmen}

Die folgenden Ausführungen beziehen sich auf die gegenwärtige Rechtslage. Sollten die für die Wearable-basierte Detektion von Arrhythmien erforderlichen Gesundheitsdaten des Patienten künftig, wie aktuell vom Reformgesetzgeber schon initiiert, mit dessen Einwilligung in eine auch im stationären Sektor 
nutzbare ePA integriert werden, werden sich die relevanten Risiken maßgeblich minimieren.

\section{Haftungsrechtliche Aspekte}

Der Einsatz von Wearables zur Detektion von Arrhythmien unterliegt denselben haftungsrechtlichen Anforderungen wie die klassische Medizin. Maßgeblich ist, ob der Arzt unter Einsatz der von ihm $\mathrm{zu}$ fordernden medizinischen Kenntnisse und Erfahrungen im konkreten Fall vertretbare Entscheidungen über die diagnostischen sowie therapeutischen Maßnahmen getroffen und diese Maßnahmen sorgfältig durchgeführt hat.

Muss der Arzt also aufgrund seiner Fachkunde erkennen können, dass die ihm via Wearable übermittelten Arrhythmiedaten im konkreten Fall falsch sind, darf er sie nicht zur Grundlage seiner Entscheidung machen. Er ist verpflichtet, die Entscheidungsgrundlage umso genauer zu prüfen, je gefährlicher sich sein Irrtum auswirkt. Dies gilt auch für erkennbare Übertragungsmängel. Unterbleibt etwa die Datenübertragung, ohne dass der Arzt reagiert, kann ein Behandlungsfehler unterstellt werden.

Für Wearables, die als Medizinprodukte zertifiziert sind, ist außerdem zu beachten, dass sie nur ihrer Zweckbestimmung entsprechend angewendet werden. Wird diese Vorgabe verletzt, kann dies unabhängig von einem persönlichen Verschulden zu einer Haftung für den entstandenen Schaden führen. Die patientenseitig generierten Daten dürfen also nur für die vom Hersteller bestimmten Zwecke verwendet werden. Andernfalls liegt ein Off-labelUse vor. Dies bedeutet, dass der behandelnde Arzt darauf achten muss, dass die Zweckbestimmung des Wearables bzw. der Software(funktionalität) auch die Information des Arztes über Arrhythmien umfasst. Die Nutzung für bestimmte diagnostische Zwecke ist auch Voraussetzung für die Zertifizierung als Medizinprodukt bzw. Medizinproduktesoftware. Soll die Applikation entsprechend der Gebrauchsanweisung Arrhythmien detektieren und diese Daten ggf. auch dem diagnostizierenden Arzt übermitteln, verbleibt die Haftung für ihr ordnungsgemäßes Funktionieren in der Sphäre des Herstellers.

\section{Datenschutzrechtliche Aspekte}

Für den Datenfluss zwischen Patient und Arzt gelten die allgemeinen Regeln des Datenschutzrechts. Das Datenschutzrecht erfasst die Verarbeitung personenbezogener Daten. Hierzu gehören auch Gesundheitsdaten, d.h. Daten, die sich auf die körperliche Gesundheit beziehen. Auf die Datenquelle kommt es nicht an. Erfasst sind also auch solche Daten, die Nutzer selbst eingeben, die durch Sensorik erfasst werden oder die eine Gesundheits-App durch Berechnungen ermittelt. Daten von Wearables über Arrhythmien sind also Gesundheitsdaten. Somit liegt eine Verarbeitung von Gesundheitsdaten vor, sobald ein Arzt ein Wearable im Rahmen einer Diagnose oder einer Therapie im weitesten Sinne einsetzt.

$\mathrm{Zu}$ beachten ist, dass für die Beurteilung ärztlicher Pflichten beim Einsatz von Wearables entscheidend ist, ob der Arzt Verantwortlicher im datenschutzrechtlichen Sinn ist. Bei Wearables, die auf Anweisung und unter Kontrolle des Arztes eingesetzt werden, ist dies der Fall. Der Arzt ist auch dann Verantwortlicher, wenn der Patient ein Wearable auf dessen Anweisung und unter dessen Kontrolle einsetzt. Zwar hat er in der Regel keine unmittelbare Kontrolle über Details der Verarbeitung, etwa die einzelnen Datenflüsse, und oft auch keinen unmittelbaren Zugriff auf die verarbeiteten Daten, entscheidet aber - und das ist aus datenschutzrechtlicher Sicht maßgebend auch über die Datenerhebung. Anders verhält es sich, wenn der Patient selbst die Verwendung des Wearables initiiert oder die generierten Daten aus eigenem Antrieb in die ePA eingibt. In diesem Fall beginnt die Verantwortlichkeit des Arztes erst mit dem Empfang der Daten bzw. mit dem ersten Zugriff auf sie oder der Einbeziehung des Wearables in die ärztliche Behandlung. Exemplarisch hierfür ist etwa die Übermittlung des Ergebnisses einer vom Patienten ohne vorherige ärztliche Anweisung selbsttätig vorgenommenen und dem Arzt per E-Mail übermittelten EKG-Messung mittels Smartwatch.
Die Datenverarbeitung ist zur Erfüllung des Behandlungsvertrages, also nicht vor dessen Abschluss, ohne Weiteres zulässig, ohne dass eine gesonderte Einwilligung eingeholt werden muss. Dazu zählt auch die Übermittlung von Patientendaten an mitbehandelnde Ärzte. Etwas anderes gilt nur dann, wenn der Arzt die Daten zu anderen Zwecken, wie etwa Forschung, verarbeiten möchte.

Verschaffen Ärzte ihrem Assistenzpersonal Zugang zu den Wearable-Daten, müssen sie darauf achten, dass die $\mathrm{Ge}$ heimhaltungsvereinbarungen auch diese Daten umfassen.

Schließlich sind u. a. auch datensicherheitsbezogene Vorgaben zu beachten. So ist die Übermittlung der Patientendaten durch nicht Ende-zu-Ende-verschlüsselte E-Mail- oder Messengerdienste zu vermeiden.

\section{Vorteile und Nachteile eines Wearables zur Arrhythmiedetektion [39]}

\section{Nachteile}

- Wearables sind für den Anwender zum Teil sehr kostenintensiv, sofern keine Erstattung durch die Krankenkassen erfolgt.

- Die Vergütung für den behandelnden Arzt ist noch nicht geklärt.

- Die Sicherheit und Verarbeitung der Patientendaten ist für den Anwender nicht immer transparent.

- Die unkontrollierte Nutzung von Wearables kann zu einer Überfrachtung an Daten führen, die durch den behandelnden Arzt nicht sinnvoll und effektiv zu handzuhaben sind.

- Patientenschulung und eine hohe Compliance sind Voraussetzungen, um mittels Wearables auf Arrhythmien zu screenen.

\section{Vorteile}

- Ein Wearable ist ohne oder mit nur wenig zusätzlicher Hardware sofort verfügbar und ermöglicht eine umgehende Aufzeichnung des Herzrhythmus.

- Wearables können als Eventrekorder und zum systematischen oder oppor- 
tunistischen Vorhofflimmerscreening eingesetzt werden.

- Wearables erlauben eine schnellere

Diagnose von Arrhythmien.

- Wearables erlauben eine stärkere

Einbindung der Patienten.

- Wearables gewährleisten eine stärkere Verbreitung diagnostischer Möglichkeiten zur Rhythmusdiagnostik.

- Einige Wearables ermöglichen ein kontinuierliches Rhythmusmonitoring.

- Die Spezifität und Sensitivität der Vorhofflimmererkennung ist $>90 \%$.

\section{Korrespondenzadresse}

Univ.-Prof. Dr. med.
Christian Veltmann
Hannover Herzrhythmus
Centrum, Klinik für
Kardiologie und Angiologie,
Medizinische Hochschule
Hannover
Carl-Neuberg-Str. 1,
30625 Hannover,
Deutschland
veltmann.christian@mh-
hannover.de

\section{Einhaltung ethischer Richtlinien}

Interessenkonflikt. Den Interessenkonflikt der Autoren finden Sie online auf der DGK-Homepage unter http://leitlinien.dgk.org/bei der entsprechenden Publikation.

Für diesen Beitrag wurden von den Autoren keine Studien an Menschen oder Tieren durchgeführt. Für die aufgeführten Studien gelten die jeweils dort angegebenen ethischen Richtlinien.

\section{Literatur}

1. Andrade JG, Godin R, Nault I (2020) Large-scale implementation of a pragmatic atrial fibrillation screening program in Canadian community practice. Pacing Clin Electrophysiol 43:768-769. https://doi.org/10.1111/pace.13966

2. Bansal A, Joshi R (2018) Portable out-of-hospital electrocardiography: a review of current technologies. J Arrhythmia 34:129-138. https://doi.org/10. 1002/joa3.12035

3. Barrett PM, Komatireddy R, Haaser S et al (2014) Comparison of 24-hour Holter monitoring with 14-day novel adhesive patch electrocardiographic monitoring. Am J Med 127(95):e11-95.e17. https://doi.org/10.1016/j.amjmed.2013.10.003

4. Behzadi A, Shamloo AS, Mouratis K et al (2020) Feasibility and reliability of smartwatch to obtain 3-lead electrocardiogram recordings. Sensors 20:5074. https://doi.org/10.3390/s20185074

5. Bonomi AG, Schipper F, Eerikäinen $L M$ et al (2018) Atrial fibrillation detection using a novel cardiac ambulatory monitor based on photoplethysmography at the wrist. J Am Heart Assoc 7:e9351. https://doi.org/10.1161/jaha.118. 009351

6. Brasier N, Raichle CJ, Dörr M et al (2018) Detection of atrial fibrillation with a smartphone camera: first prospective, international, two-centre, clinical validation study (DETECT AF PRO). Europace 21:41-47. https://doi.org/10.1093/europace/ euy 176

7. Castelletti S, Dagradi F, Goulene K et al (2018) A wearable remote monitoring system for the identification of subjects with a prolonged QT interval or at risk for drug-induced long QT syndrome. Int JCardiol 266:89-94. https://doi.org/ 10.1016/j.ijcard.2018.03.097

8. Chan P, Wong C, Poh YC et al (2016) Diagnostic performance of a smartphone-based photoplethysmographic application for atrial fibrillation screening in a primary care setting. J Am Heart Assoc. https://doi.org/10.1161/jaha.116.003428

9. Choi W, Kim S-H, Lee W et al (2020) Comparison of continuous ECG monitoring by wearable patch device and conventional telemonitoring device. JKorean Med Sci 35:e363. https://doi.org/10.3346/ jkms.2020.35.e363

10. Couderc J-P, Kyal S, Mestha LKetal (2015) Detection of atrial fibrillation using contactless facial video monitoring. Heart Rhythm 12:195-201. https:// doi.org/10.1016/j.hrthm.2014.08.035

11. Diederichsen SZ, Haugan KJ, Kronborg C et al (2020) Comprehensive evaluation of rhythm monitoring strategies in screening for atrial fibrillation. Circulation 141:1510-1522. https:// doi.org/10.1161/circulationaha.119.044407

12. Ding EY, Svennberg E, Wurster C et al (2020) Survey of current perspectives on consumeravailable digital health devices for detecting atrial fibrillation. Cardiovasc Digit Health J 1:21-29. https://doi.org/10.1016/j.cvdhj.2020.06.002

13. Duncker D, Ding WY, Etheridge S et al (2021) Smart wearables for cardiac monitoring - real-world use beyond atrial fibrillation. Sensors 21:2539. https:// doi.org/10.3390/s21072539

14. Eerikinen LM, Bonomi AG, Schipper F et al (2019) Detecting atrial fibrillation and atrial flutter in daily life using photoplethysmography data. IEEE J Biomed Health Inform 24:1610-1618. https://doi. org/10.1109/jbhi.2019.2950574

15. Elgendi M, Eskofier B, Dokos S, Abbott D (2014) Revisiting QRS detection methodologies for portable, wearable, battery-operated, and wireless ECG systems. PLoS One 9:e84018. https://doi.org/ 10.1371/journal.pone.0084018

16. Fallet $S$, Lemay M, Renevey $P$ et al (2019) Can one detect atrial fibrillation using a wrist-type photoplethysmographic device? Med Biol Eng Comput 57:477-487. https://doi.org/10.1007/ s11517-018-1886-0

17. Fouassier D, Roy X, Blanchard A, Hulot J (2020) Assessment of signal quality measured with a smart 12-lead ECG acquisition T-shirt. Ann Noninvasive Electrocardiol 25:e12682. https://doi. org/10.1111/anec.12682

18. Freedman B, Schnabel R, Calkins H (2019) Opportunistic electrocardiogram screening for atrial fibrillation to prevent stroke. JAMA Cardiol 4:54-55. https://doi.org/10.1001/jamacardio. 2018.4335
19. Fukuma N, Hasumi E, Fujiu Ket al (2019) Feasibility of a T-shirt-type wearable electrocardiography monitor for detection of covert atrial fibrillation in young healthy adults. Sci Rep 9:11768. https://doi. org/10.1038/s41598-019-48267-1

20. Gawalko M, Duncker D, Manninger M et al (2021) The European TeleCheck-AF project on remote app-based management of atrial fibrillation during the COVID-19 pandemic: centre and patient experiences. Europace. https://doi.org/10.1093/ europace/euaa394

21. Gillinov S, Etiwy M, Wang Ret al (2017) Variable accuracy of wearable heart rate monitors during aerobic exercise. Med Sci Sports Exerc 49:1697-1703. https://doi.org/10.1249/mss.0000000000001284

22. Godin R, Yeung C, Baranchuk A et al (2019) Screening for atrial fibrillation using a mobile, singlelead electrocardiogram in Canadian primary care clinics. Can J Cardiol 35:840-845. https://doi.org/ 10.1016/j.cjca.2019.03.024

23. Goldenthal IL, Sciacca RR, Riga T et al (2019) Recurrent atrial fibrillation/flutter detection after ablation or cardioversion using the AliveCor KardiaMobile device: iHEART results. J Cardiovasc Electrophysiol 30:2220-2228. https://doi.org/10. 1111/jce.14160

24. Guo $Y$, Wang H, Zhang $H$ et al (2019) Mobile photoplethysmographic technology to detect atrial fibrillation. J Am Coll Cardiol 74:2365-2375. https://doi.org/10.1016/j.jacc.2019.08.019

25. Haberman ZC, Jahn RT, Bose R et al (2015) Wireless smartphone ECG enables large-scale screening in diverse populations. J Cardiovasc Electrophysiol 26:520-526. https://doi.org/10.1111/jce.12634

26. Halcox JPJ, Wareham K, Cardew A et al (2017) Assessment of remote heart rhythm sampling using the AliveCor heart monitor to screen for atrial fibrillation. Circulation 136:1784-1794. https:// doi.org/10.1161/circulationaha.117.030583

27. Han JK, Al-Khatib SM, Albert CM (2020) Changes in the digital health landscape in cardiac electrophysiology: a pre- and peri-pandemic COVID-19 era survey. Cardiovasc Digit Health J. https://doi.org/ 10.1016/j.cvdhj.2020.12.001

28. Heo NJ, Rhee SY, Waalen J, Steinhubl S (2020) Chronic kidney disease and undiagnosed atrial fibrillation in individuals with diabetes. Cardiovasc Diabetol 19:157. https://doi.org/10.1186/s12933020-01128-y

29. Hermans ANL, Velden RMJ, Gawalko Met al (2020) On-demand mobile health infrastructures to allow comprehensive remote atrial fibrillation and risk factor management through teleconsultation. Clin Cardiol 43:1232-1239. https://doi.org/10.1002/ clc.23469

30. Hindricks G, Potpara T, Dagres N et al (2020) 2020 ESC guidelines for the diagnosis and management of atrial fibrillation developed in collaboration with the European association of cardio-thoracic surgery (EACTS). Eur Heart J. https://doi.org/10. 1093/eurheartj/ehaa612

31. Jaakkola J, Virtanen R, Vasankari T et al (2017) Self-detection of atrial fibrillation in an aged population: three-year follow-up of the LietoAF intervention study. BMC Geriatr 17:218. https:// doi.org/10.1186/s12877-017-0607-0

32. Kaleschke G, Hoffmann B, Drewitz I et al (2009) Prospective, multicentre validation of a simple, patient-operated electrocardiographic system for the detection of arrhythmias and electrocardiographic changes. Europace 11:1362-1368. https:// doi.org/10.1093/europace/eup262

33. Kearley K, Selwood M, den Bruel AV et al (2014) Triage tests for identifying atrial fibrillation 
in primary care: a diagnostic accuracy study comparing single-lead ECG and modified BP monitors. BMJ Open 4:e4565. https://doi.org/10. 1136/bmjopen-2013-004565

34. Koshy AN, Sajeev JK, Nerlekar N et al (2018) Smart watches for heart rate assessment in atrial arrhythmias. Int JCardiol 266:124-127. https://doi. org/10.1016/j.ijcard.2018.02.073

35. Lau JK, Lowres N, Neubeck Letal (2013) iphone ECG application for community screening to detect silent atrial fibrillation: a novel technology to prevent stroke. Int J Cardiol 165:193-194. https:// doi.org/10.1016/j.ijcard.2013.01.220

36. Li X, Tse VC, Au-Doung LW et al (2016) The impact of ischaemic stroke on atrial fibrillation-related healthcare cost: a systematic review. Europace. https://doi.org/10.1093/europace/euw093

37. Lowres N, Mulcahy G, Gallagher Ret al (2016) Selfmonitoring for atrial fibrillation recurrence in the discharge period post-cardiac surgery using an iPhone electrocardiogram. Eur J Cardiothorac Surg 50:44-51.https://doi.org/10.1093/ejcts/ezv486

38. Lowres N, Neubeck L, Salkeld G et al (2014) Feasibility and cost-effectiveness of stroke prevention through community screening for atrial fibrillation using iPhone ECG in pharmacies. Thromb Haemost 111:1167-1176. https://doi.org/10.1160/th1403-0231

39. Manninger M, Kosiuk J, Zweiker D et al (2020) Role of wearable rhythm recordings in clinical decision making - the wEHRables project. Clin Cardiol 43:1032-1039. https://doi.org/10.1002/clc.23404

40. Manninger $M$, Zweiker $D$, Svennberg $E$ et al (2021) Current perspectives on wearable rhythm recordings for clinical decision-making: the wEHRAbles 2 survey. Europace. https://doi.org/10. 1093/europace/euab064

41. McManus DD, Lee J, Maitas O et al (2013) A novel application for the detection of an irregular pulse using an iPhone 45 in patients with atrial fibrillation. Heart Rhythm 10:315-319. https://doi. org/10.1016/j.hrthm.2012.12.001

42. Muhlestein JB, Anderson JL, Bethea CF et al (2020) Feasibility of combining serial smartphone single-lead electrocardiograms for the diagnosis of ST-elevation myocardial infarction. Am Heart J 221:125-135. https://doi.org/10.1016/j.ahj.2019. 12.016

43. Murali S, Brugger N, Rincon F et al (2020) Cardiac ambulatory monitoring: new wireless device validated against conventional Holter monitoring in a case series. Front Cardiovasc Med 7:587945. https://doi.org/10.3389/fcvm.2020.587945

44. Nemati S, Ghassemi MM, Ambai V et al (2016) Monitoring and detecting atrial fibrillation using wearable technology. Annu Int Conf IEEE Eng Med Biol Soc 2016:3394-3397. https://doi.org/10. 1109/embc.2016.7591456

45. Nguyen HH, Hare GFV, Rudokas M et al (2015) SPEAR trial: smartphone pediatric ElectrocARdiogram trial. PLoS ONE 10:e136256. https://doi.org/ 10.1371/journal.pone.0136256

46. Orchard J, Lowres N, Freedman SB et al (2016) Screening for atrial fibrillation during influenza vaccinations by primary care nurses using a smartphone electrocardiograph (iECG): a feasibility study. Eur J Prev Cardiol 23:13-20. https://doi.org/10.1177/2047487316670255

47. Paradkar N, Chowdhury SR (2017) Cardiac arrhythmia detection using photoplethysmography. Annu Int Conf IEEEEng Med Biol Soc 2017:113-116. https://doi.org/10.1109/embc.2017.8036775

48. Perez MV, Mahaffey KW, Hedlin Hetal (2019) Largescale assessment of a smartwatch to identify atrial fibrillation. N Engl J Med 381:1909-1917. https:// doi.org/10.1056/nejmoa1901183

49. Pluymaekers NAHA, Hermans ANL, van der Velden RMJ et al (2020) Implementation of an on-demand app-based heart rate and rhythm monitoring infrastructure for the management of atrial fibrillation through teleconsultation: TeleCheck-AF. Europace. https://doi.org/10.1093/ europace/euaa201

50. Polania LF, Mestha LK, Huang DT, Couderc J-P (2015) Method for classifying cardiac arrhythmias using photoplethysmography. Annu Int Conf IEEE Eng Med Biol Soc 2015:6574-6577. https://doi. org/10.1109/embc.2015.7319899

51. Proesmans T, Mortelmans C, Haelst RV et al (2019) Mobile phone-based use of the photoplethysmography technique to detect atrial fibrillation in primary care: diagnostic accuracy study of the Fibricheck app. JMIR Mhealth Uhealth 7:e12284. https://doi.org/10.2196/12284

52. Quer G, Freedman B, SteinhubISR (2020) Screening for atrial fibrillation: predicted sensitivity of short, intermittent electrocardiogram recordings in an asymptomatic at-risk population. Europace 22:euaa186. https://doi.org/10.1093/europace/ euaa186

53. Rosenberg MA, Samuel M, Thosani A, Zimetbaum PJ (2013) Use of a noninvasive continuous monitoring device in the management of atrial fibrillation: a pilot study. Pacing Clin Electrophysio 36:328-333. https://doi.org/10.1111/pace.12053

54. Samol A, Bischof K, Luani B et al (2019) Singlelead ECG recordings including Einthoven and wilson leads by a smartwatch: a new era of patient directed early ECG differential diagnosis of cardiac diseases? Sensors 19:4377. https://doi.org/10. 3390/s19204377

55. Sarkar S, Ritscher D, Mehra R (2008) A detector for a chronic implantable atrial tachyarrhythmia monitor. IEEE Trans Biomed Eng 55:1219-1224. https://doi.org/10.1109/tbme.2007.903707

56. Scholz EP, Kehrle F, Vossel S et al (2014) Discriminating atrial flutter from atrial fibrillation using a multilevel model of atrioventricular conduction. Heart Rhythm 11:877-884. https://doi.org/10. 1016/j.hrthm.2014.02.013

57. Siontis KC, Gersh BJ, Killian JM et al (2016) Typical, atypical, and asymptomatic presentations of new-onset atrial fibrillation in the community: characteristics and prognostic implications. Heart Rhythm 13:1418-1424. https://doi.org/10.1016/j. hrthm.2016.03.003

58. Sološenko A, Petrènas A, Marozas V (2015) Photoplethysmography-based method for automatic detection of premature ventricular contractions. IEEE Trans Biomed Circuits Syst 9:662-669. https:// doi.org/10.1109/tbcas.2015.2477437

59. Spaich S, Kern H, Zelniker TA et al (2020) Feasibility of CardioSecur ${ }^{\circledR}$, a mobile 4-electrode/22-lead ECG device, in the prehospital emergency setting Front Cardiovasc Med 7:551796. https://doi.org/ 10.3389/fcvm.2020.551796

60. Steinberg C, Philippon F, Sanchez M et al (2019) A novel wearable device for continuous ambulatory ECG recording: proof of concept and assessment of signal quality. Biosensors 9:17. https://doi.org/10. 3390/bios9010017

61. Taggar JS, Coleman T, Lewis Set al (2015) Accuracy of methods for detecting an irregularpulseand suspected atrial fibrillation: a systematic review and meta-analysis. Eur J Prev Cardiol 23:1330-1338. https://doi.org/10.1177/2047487315611347

62. Tison GH, Sanchez JM, Ballinger B et al (2018) Passive detection of atrial fibrillation using a com- mercially available smartwatch. JAMA Cardiol 3:409. https://doi.org/10.1001/jamacardio.2018. 0136

63. Turakhia MP, Hoang DD, Zimetbaum P et al (2013) Diagnostic utility of a novel leadless arrhythmia monitoring device. Am J Cardiol 112:520-524. https://doi.org/10.1016/j.amjcard.2013.04.017

64. Varma N, Marrouche NF, Aguinaga L et al (2020) HRS/EHRA/APHRS/LAHRS/ACC/AHA worldwide practice update for telehealth and arrhythmia monitoring during and after a pandemic. Europace 23:euaa187. https://doi.org/10.1093/europace/ euaa187

65. Verbrugge $\mathrm{FH}$, Proesmans T, Vijgen J et al (2019) Atrial fibrillation screening with photoplethysmography through a smartphone camera. Europace 21:1167-1175. https://doi.org/10.1093/ europace/euz119

66. Wang R, Blackburn G, Desai Met al (2016) Accuracy of wrist-worn heart rate monitors. JAMA Cardiol 2:104. https://doi.org/10.1001/jamacardio.2016. 3340

67. Yan BP, Lai WHS, Chan CKY et al (2018) Contactfree screening of atrial fibrillation by a smartphone using facial pulsatile photoplethysmographic signals. J Am Heart Assoc. https://doi.org/10.1161/ jaha.118.008585

68. Yan BP, Lai WHS, Chan CKY et al (2020) Highthroughput, contact-free detection of atrial fibrillation from video with deep learning JAMA Cardiol 5:105-107. https://doi.org/10.1001/ jamacardio.2019.4004

69. Zaprutko T, Zaprutko J, Baszko A et al (2019) Feasibility of atrial fibrillation screening with mobile health technologies at pharmacies. J Cardiovasc Pharmacol Ther 25:142-151. https:// doi.org/10.1177/1074248419879089

70. Zink MD, Mischke KG, Keszei AP et al (2020) Screendetected atrial fibrillation predicts mortality in elderly subjects. Europace 23:euaa190. https://doi. org/10.1093/europace/euaa190 